A REVIEW OF COMPUTER AIDED INTERPRETATION TECHNOLOGY FOR THE

EVALUATION OF RADIOGRAPHS OF ALUMINUM WELDS

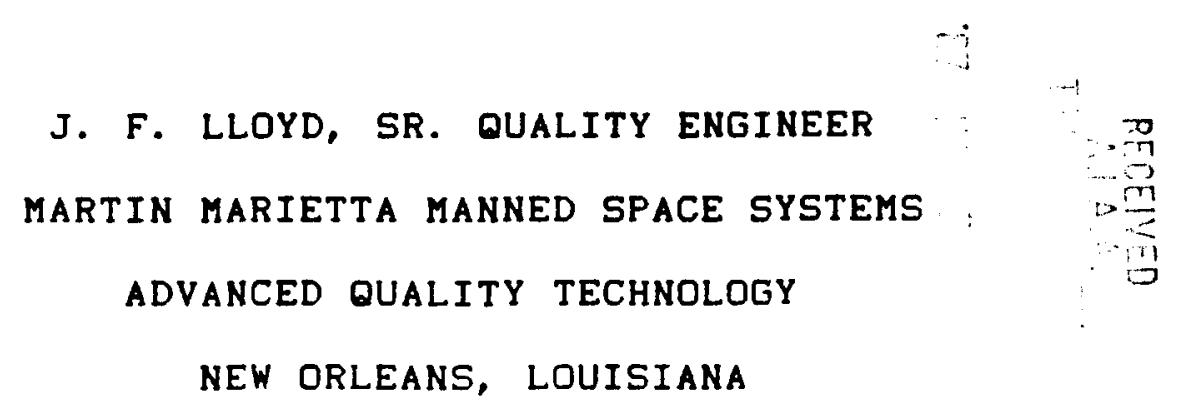

ABSTRACT: Industrial radiography is a well established, reliable means of providing nondestructive structural integrity information. The majority of industrial radiographs are interpreted by trained human eyes using transmitted light and various visual aids. Hundreds of miles of radiographic information are evaluated, documented and archived annually. In many instances, there are serious considerations in terms of interpreter fatigue, subjectivity and limited archival space. Quite often it is difficult to quickly retrieve radiographic information for further analysis or investigation. Methods of improving the quality and efficiency of the radiographic process are being explored, developed and incorporated whenever feasible. High resolution cameras, digital image processing, and mass digital data storage offer interesting possibilities for improving the industrial radiographic process.

This paper will present a review of computer alded radiographic interpretation technology in terms of how it could be used to enhance the radiographic interpretation process in evaluating radlographs of aluminum welds.

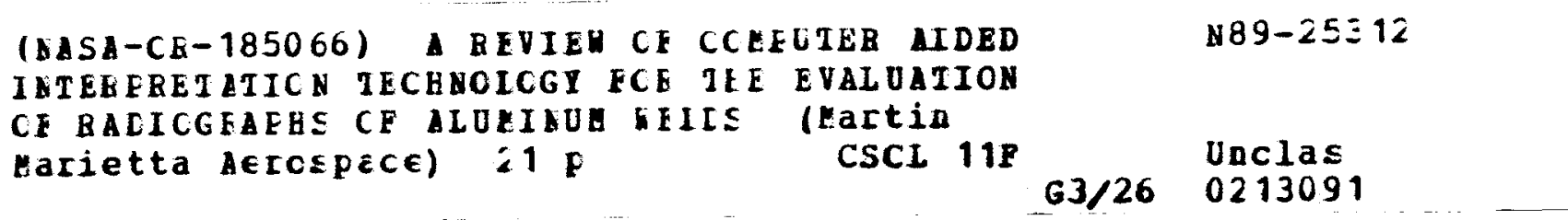


INTRODUCTION: Radiographic film interpretation, evaluation and archiving are time consuming, laborious and expensive, operations.

There is a definite need to reduce operator fatigue, subjectivity of interpretation, storage space and inspection time. In responge to that need Martin MarAjtta Manned Space Systems has been surveying components for integration into a computer alded radiographic film interpretation system for use in evaluation of aluminum welds. Components being evaluated are: high resolution cameras, digital image processing, optical disk storage and volce recognition.

\section{SURVEY RESULTS:}

CAMERAS: The results of an inspection process are only as good as the weakest link in the system. Of primary importance is the basic detector, the camera, which in effect must match or exceed the capability of the human eye. There are several cameras available in both the Vidicon and CCD categories which show acceptable image contrast and detall sensitivity detection capabilities. See figure 1 for contrast and detall sensitivity in radiograph of penetrameter.

Figure 2 shows a Vidicon and a CCD camera. For this discussion Vidicon cameras have been generalized as cameras employing electron tube type sensors. Vidicons represent a mature technology with improvements coming at a slower rate. They are generally larger. CCD cameras have been generalized as cameras employing solid state image sensors. They represent a rapidly advancing technology. They are generally smaller and more rugged. Each has its own advantages and disadvantsges. 
The Vidicon camera's horizontal resolution is determined by the video amplifier's bandwidth and the quality of construction of the vidicon itself. The vertical resolution is determined by the number of horizontal scan lines. The video signal of the vidicon camera is relatively noise free and follows the imaged object in a continuous fashion, thus the sampling analog-to-digital converter (A/D) can readily plck up a representative digital image. The vidicon though is susceptible to some extent to external magnetic fields, blooming and lag, and may suffer permanent damage from burning an image into its photosensitive surface.

The CCD camera on the other hand is much more immune to image burning and blooming. Lag and magnetic flelds are not problems. Its resolution is fixed by the geometry of the CCD chip array. The specification of this resolution and the actual number of photosites contributing to the picture sometimes appear in conflict. Care must be taken when interpreting this specification. The video signal of the CCD camera can contain a significant amount of clock noise in the time between actual pixel element signals. The sampling $A / D$ must be synchronized to pixel rate and in the proper phase relationship or the clock noise will appear in the digitized image.

Figure 3 shows a temporary setup of camera and radiograph viewer. Proper selection of a camera and lens will depend on application requirements. Camera costs can range from $\$ 2,000$ to \$90,000. CCD camera costs go to both price extremes while Vidicon prices fall into a smaller range near the low extreme. When balancing system requirements with equipment available cost is a sizable factor to be considered. We found that a vidicon with 1600 TV lines and a CCD with $1024 \times 512$ elements were adequate for our requirements. 
MONITORS: Selection of a monitor depends on application requirements and must complement the rest of the system. A high resolution black and white monitor is sufficlent for viewing original or enhanced radiographic images since there is no color information in the original radiograph. However, a high resolution RGB color monitor allows pseudo coloring which can be used to sharply distinguish very slight intensity changes. It should be noted that room illumination and brightness and contrast settings have a great effect on the apparent quality of the monitor.

IMAGE PROCESSING: Figure 4 shows an image processor used as part of a real time radiography system. Gray scale resolution and pixel resolution are basic considerations of an image processing system. Eight bits per pixel gray scale resolution is a reasonable choice for digitization and storage of images. While a higher number of bits appear better, the advantages would be in large part offset by increased storage requirements and increased noise plckup from the camera. There are dozens of image processing algorithms available to process and enhance data. ( Figure 5 shows a frame averaged image of a weld flaw.) Which ones to use depends on their particular effect on the information required. There are many usable filters which can improve the 'seeability' of flaw indications in radiographs of welds. Examples are: Laplacian, Sobel, Roberts, median, homomorphic filters, histogram equilization and neighborhood averaging. Other utilities such as zoom, scroll, pan, histograms, densitometric measurements, and micrometer capabilities are also available. Additional information on typical image processing operations is given in Appendix 1.

LASER DISK STORAGE: Since the digitization and storage of the resulting data amounts to many gigabytes of digital information, the archival mechanism should be compact and readily accessible. 
Figure 6 shows a representative laser optical disk storage subsystem. Laser optical disks have the capability of storing 2-gigabytes of digital information (1-gigabyte per side). Other smaller versions are also available. Even though 2 -gigabytes capacity appears to be large, 1 t is quite likely that various forms of data compression would be needed to further increase storage density depending on the quantity of film to be eveluated and stored. Laser disk storage also offers the advantage of . 5 second recall time per frame of information. Film archival storage consumes a lot of space as shown by figure 7. Additional time is consumed in retrival by having to search serially through many feet of film to get to the section of interest.

VOICE RECOGNITION: Initial surveys indicate that volce recognition could be used advantageously to command a considerable portion of system operation. Figure 8 shows a representative voice recognition subsystem. Verbally entered evaluations and other pertinent information could be archieved together with the actual digital information of the radiograph. More evaluation of real-world capabilities is necessary for a more definitive understanding of the voice recognition capabilities.

COMPUTER AND PERIPHERALS: Figure 9 shows a laboratory setup. of image processor, camera, film, and viewer. There are several computers which would accomplish the requirements in a film Iriterpretation system. The computer eystem arrangemente for $1 \mathrm{mag}$ processing can vary considerably. They can use general purpose micro, mini, or mainframe computers with image processing software or they can use special array and pipelined processor hardware under the orchestration of a general purpose computer. Others can be hardwired and hardcoded to perform a series of enhancement functions that are very fast but inflexible. 
CONCLUSIONS: A computer aided radiographic interpretation system shows considerable promise as a viable tool to enhance the radiographic film intrepretation process for aluminum welds. The major components for such a system have been 1dentified. They are high resolution cameras and monitors, image enhancement hardware and software, laser disk storage and volce recognition subsystems. While many of these components and systems have been evaluated, additional evaluation and data gathering are necessary. Toward this end, evaluation of a turn-key system on production film is planned for the near future. 


\section{7 of 7}

LIST OF FIGURES:

F1g. 1 Detall and contrast sensitivity of penetrameter.

Fig. 2 Vidicon and CCD cameras.

Fig. 3 Camera and viewer.

Fig. 4 Advent II Image Processor.

Fig. 5 Weld flaw on monitor using frame averaging.

F1g. 6 Laser Optical Disk Storage literature.

Fig. 7 Film Cannister Archive.

Fig. 8 Voice Recognition literature.

Fig. 9 Image Processor, Camera, F1lm and Viewer.

\section{APPENDIX \#1 Typical Image Processing Operations}




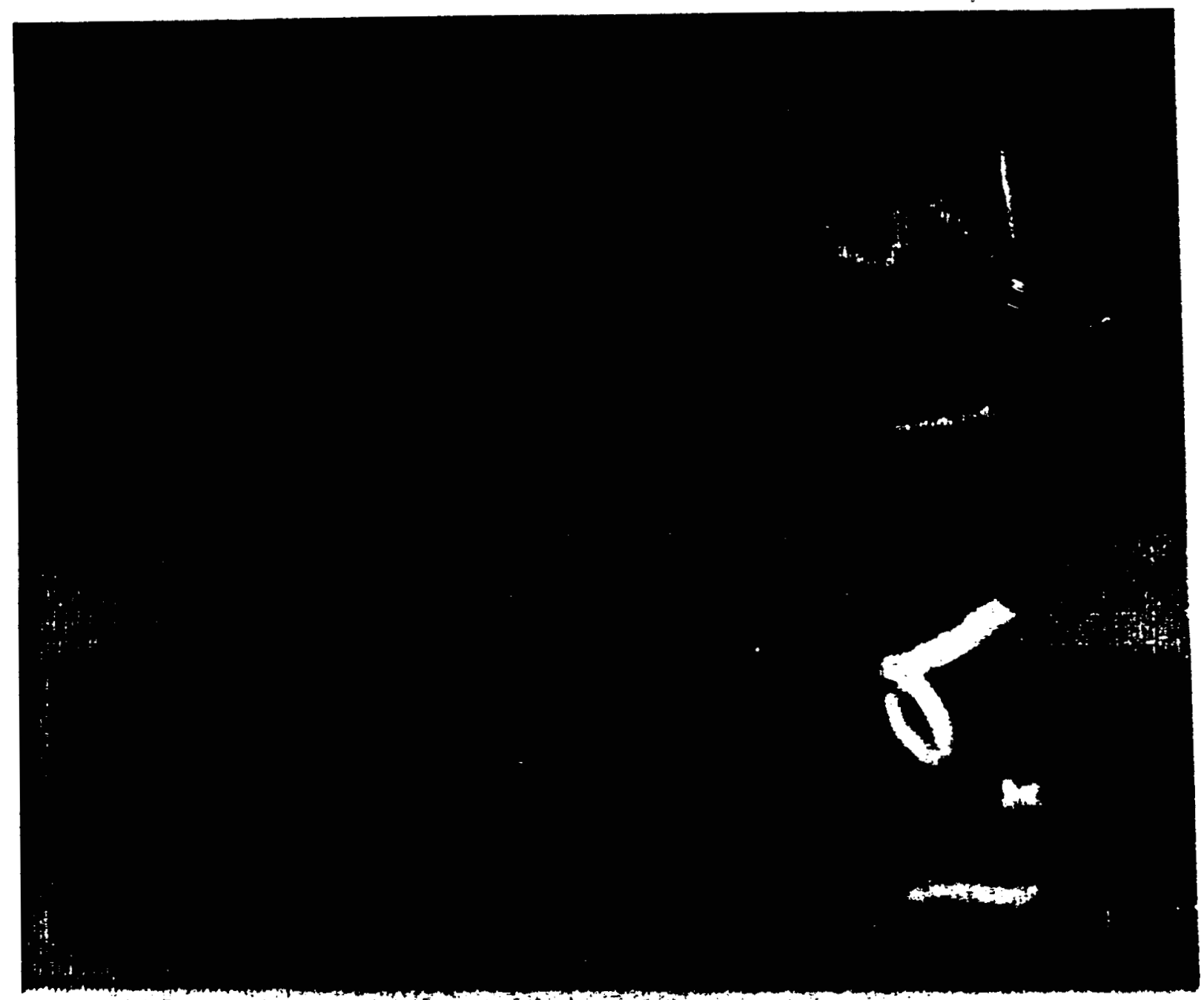

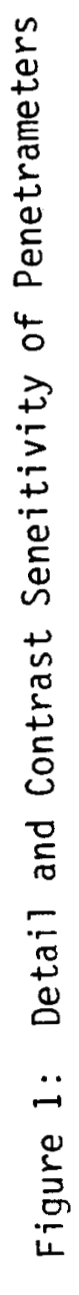
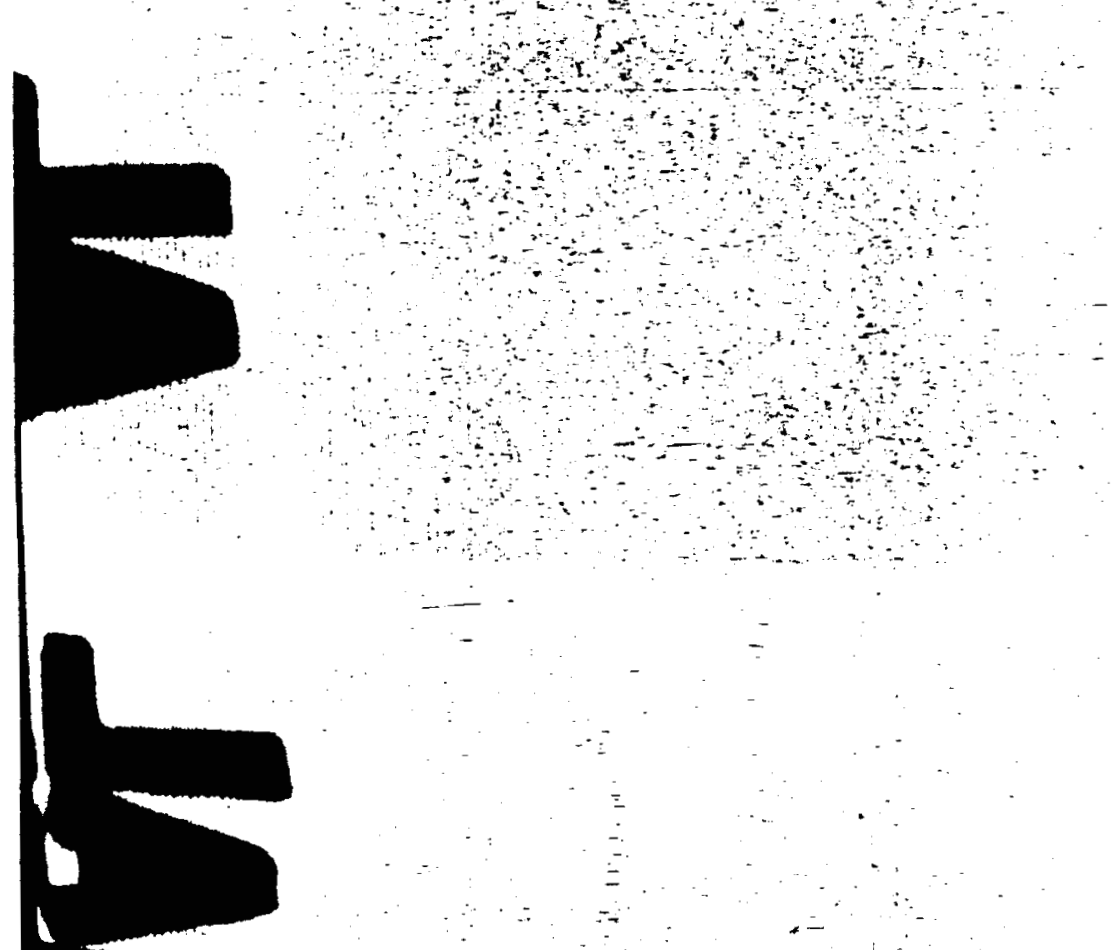


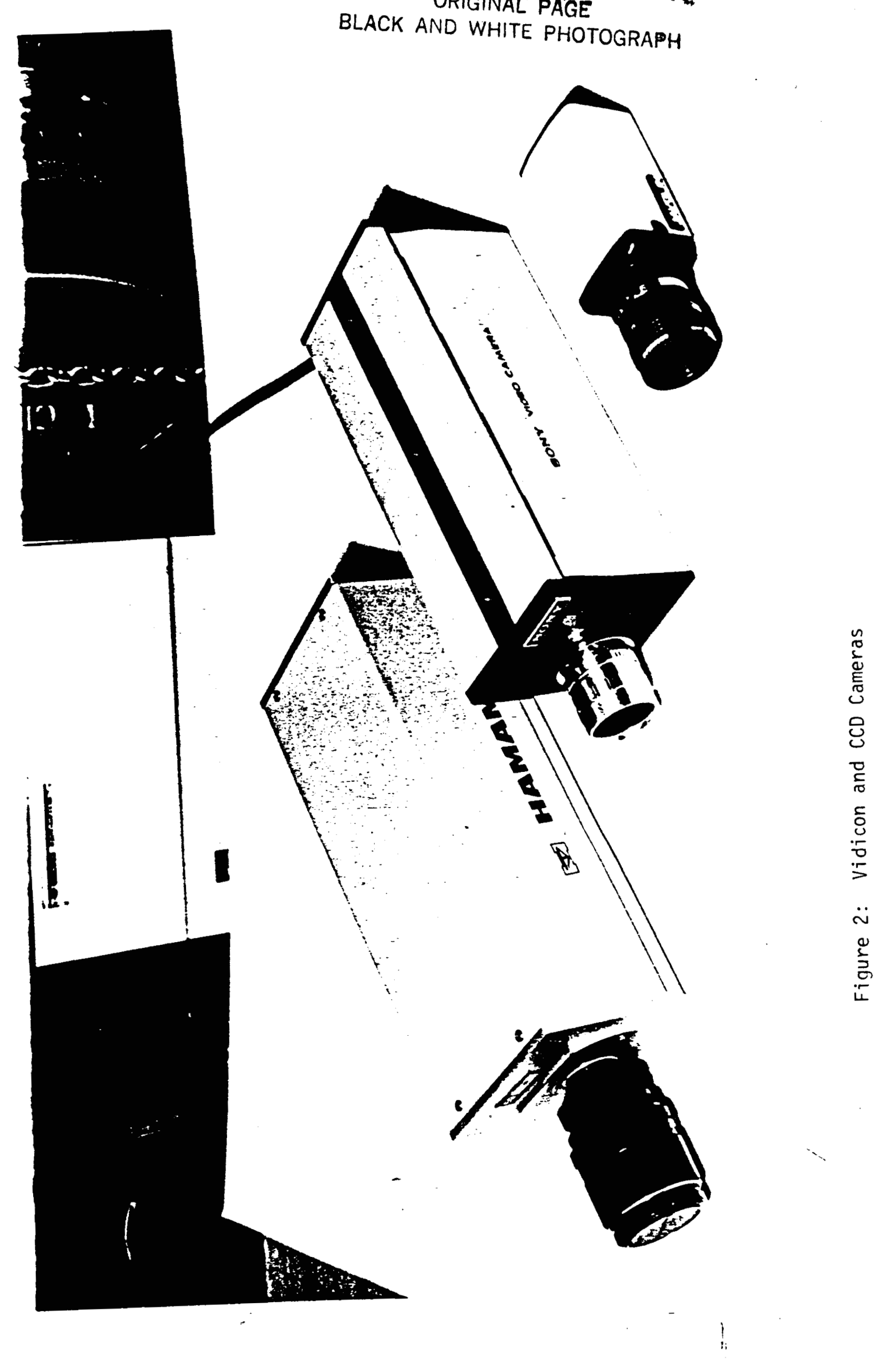


‘) H. s. (n) if ing ift. Aftit?

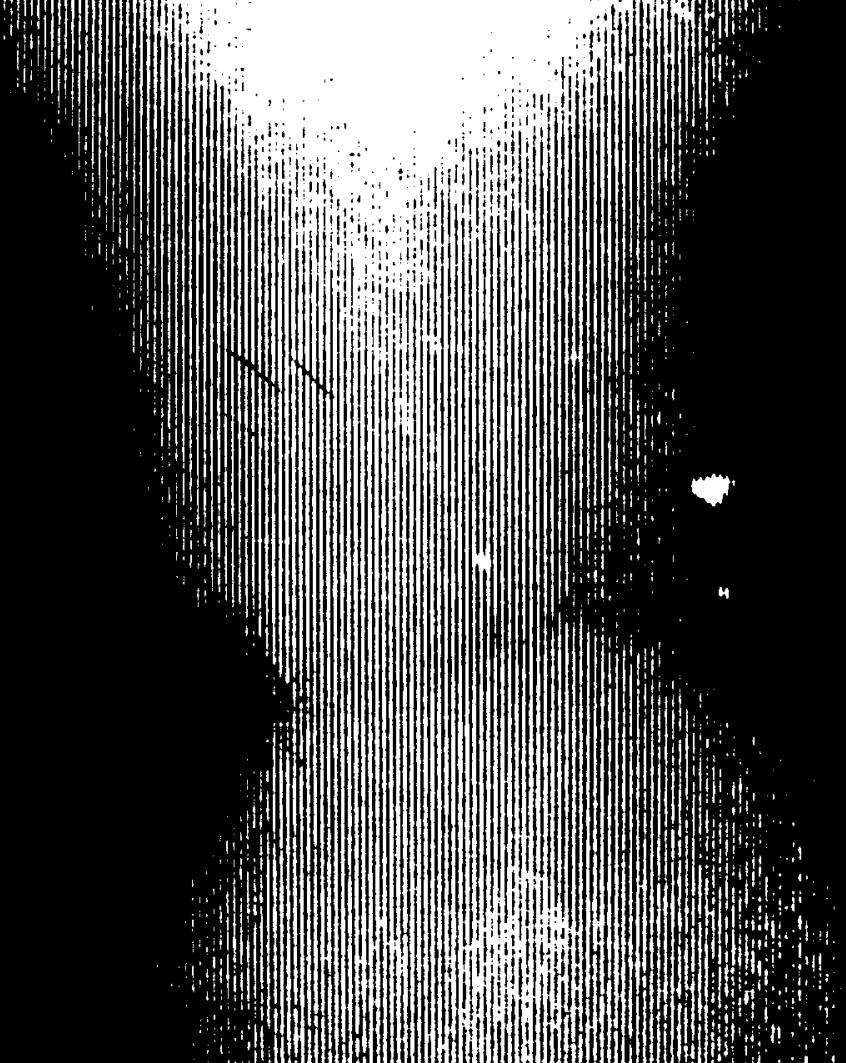
(n) 1,2, 然 :

:

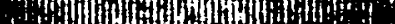

, A

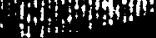




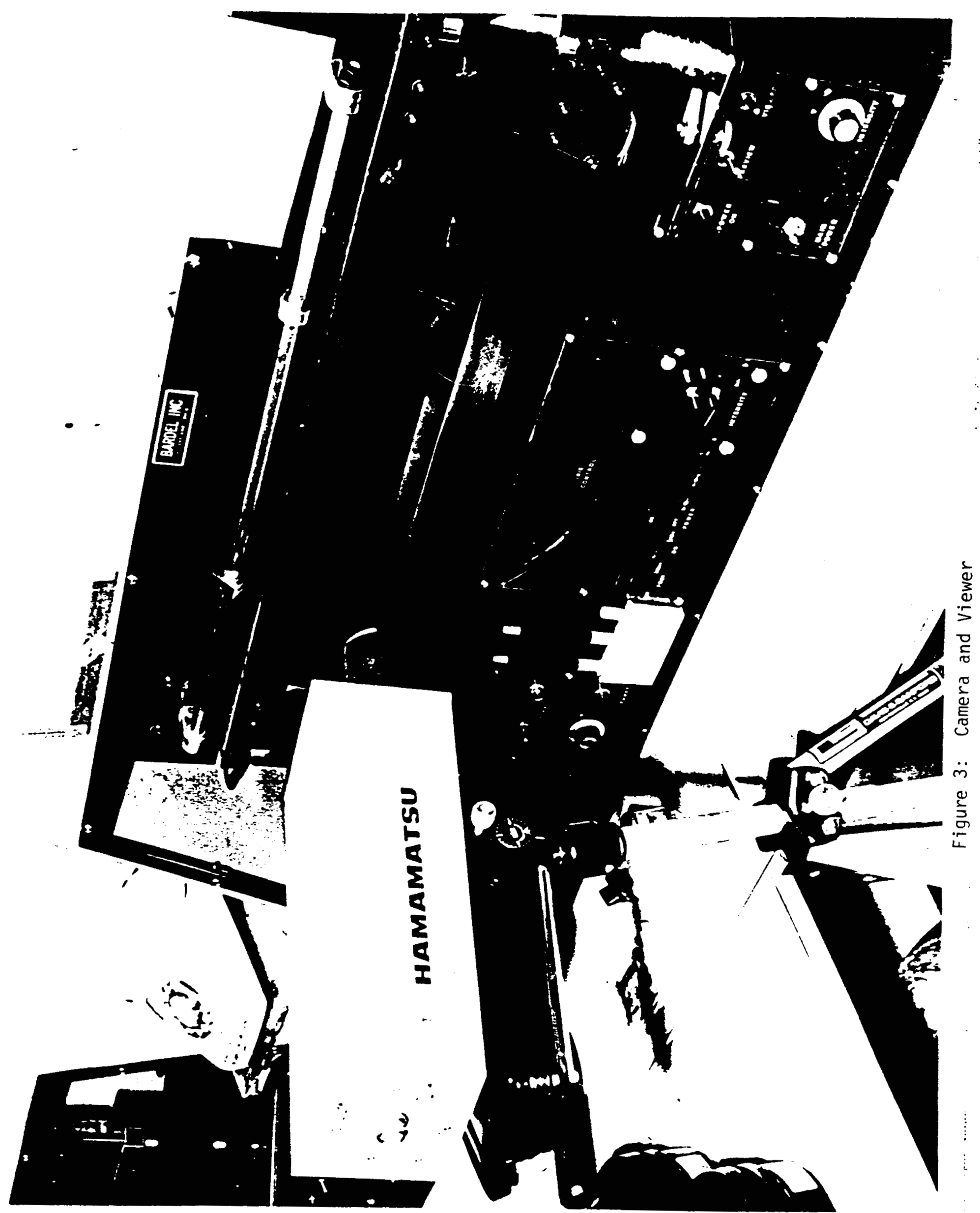




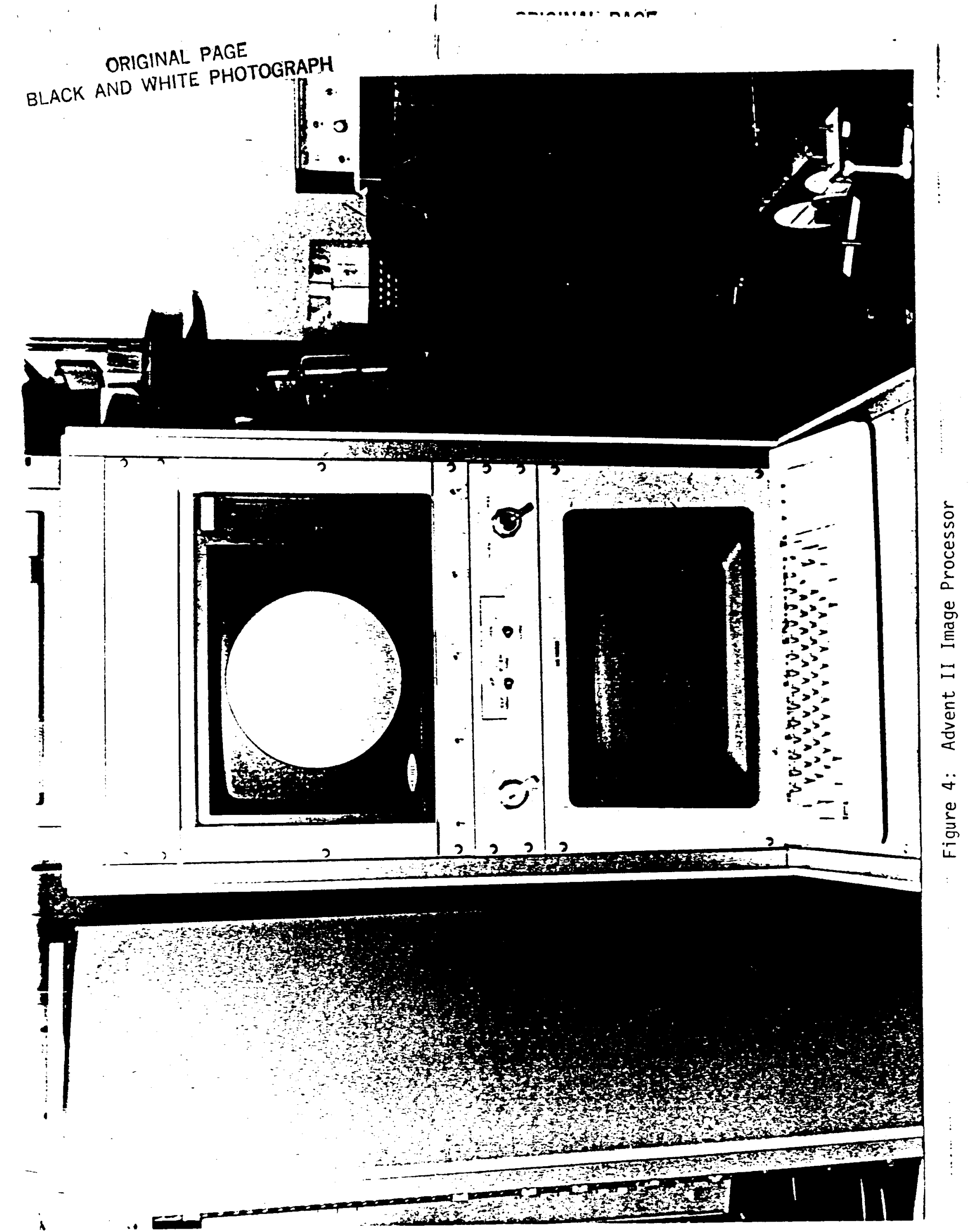


14tom

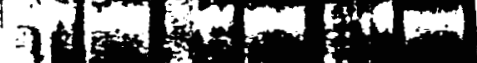

票

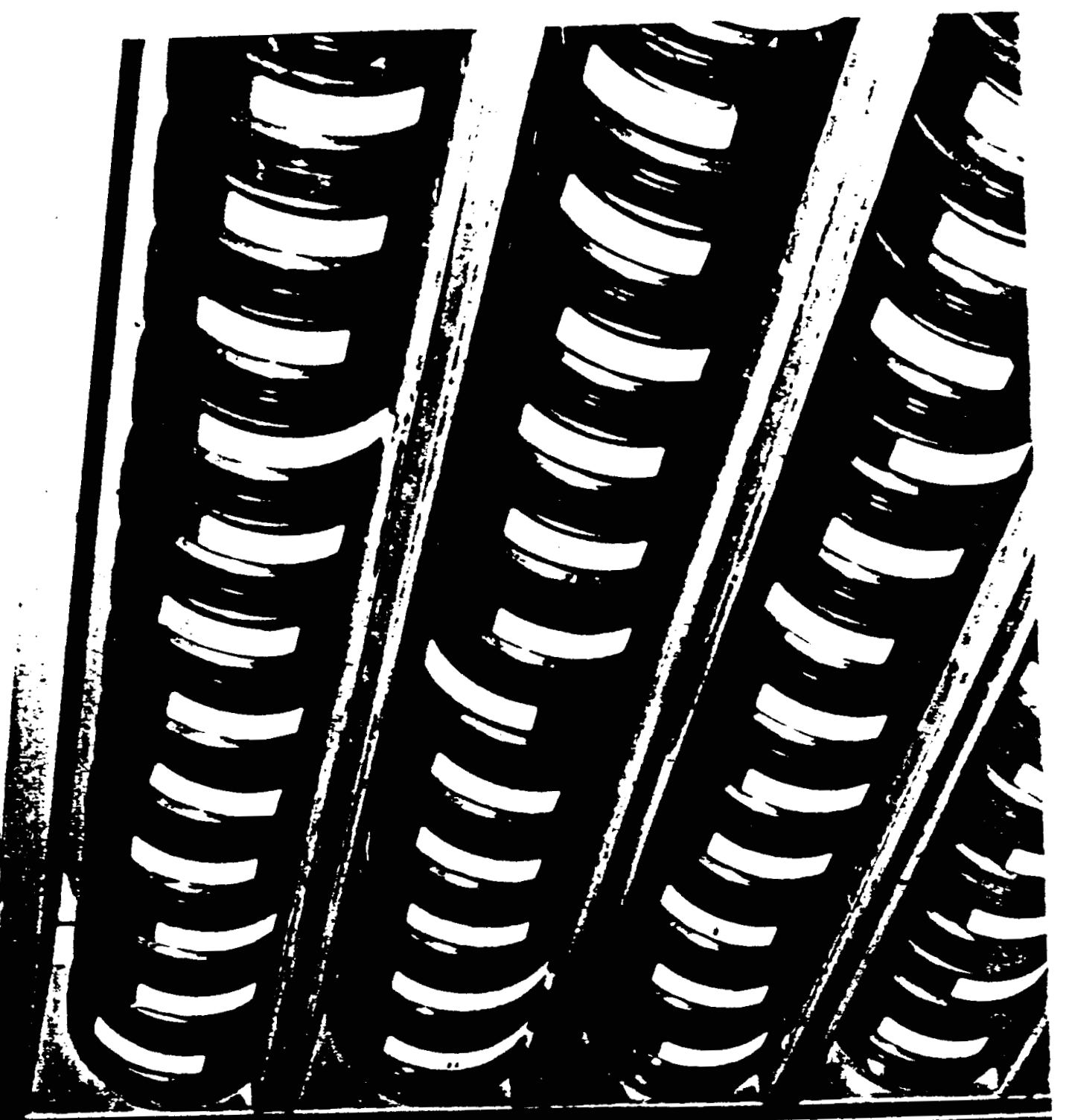
tiam 19 Inima and

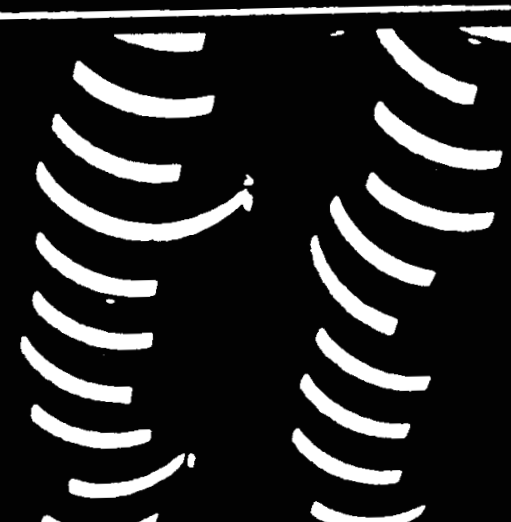




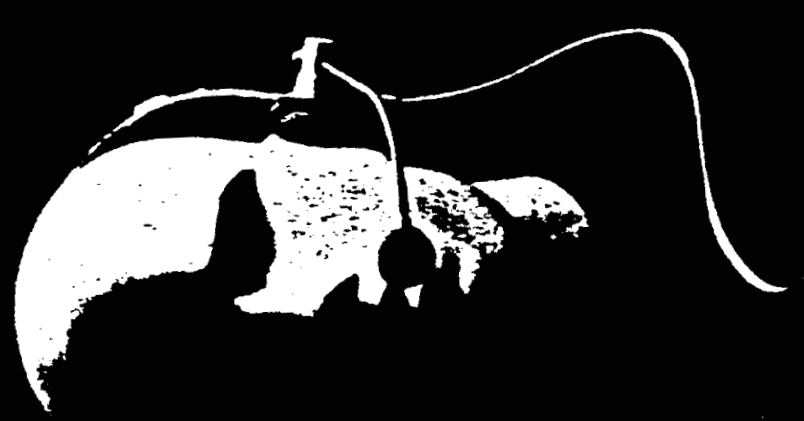

1

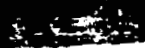
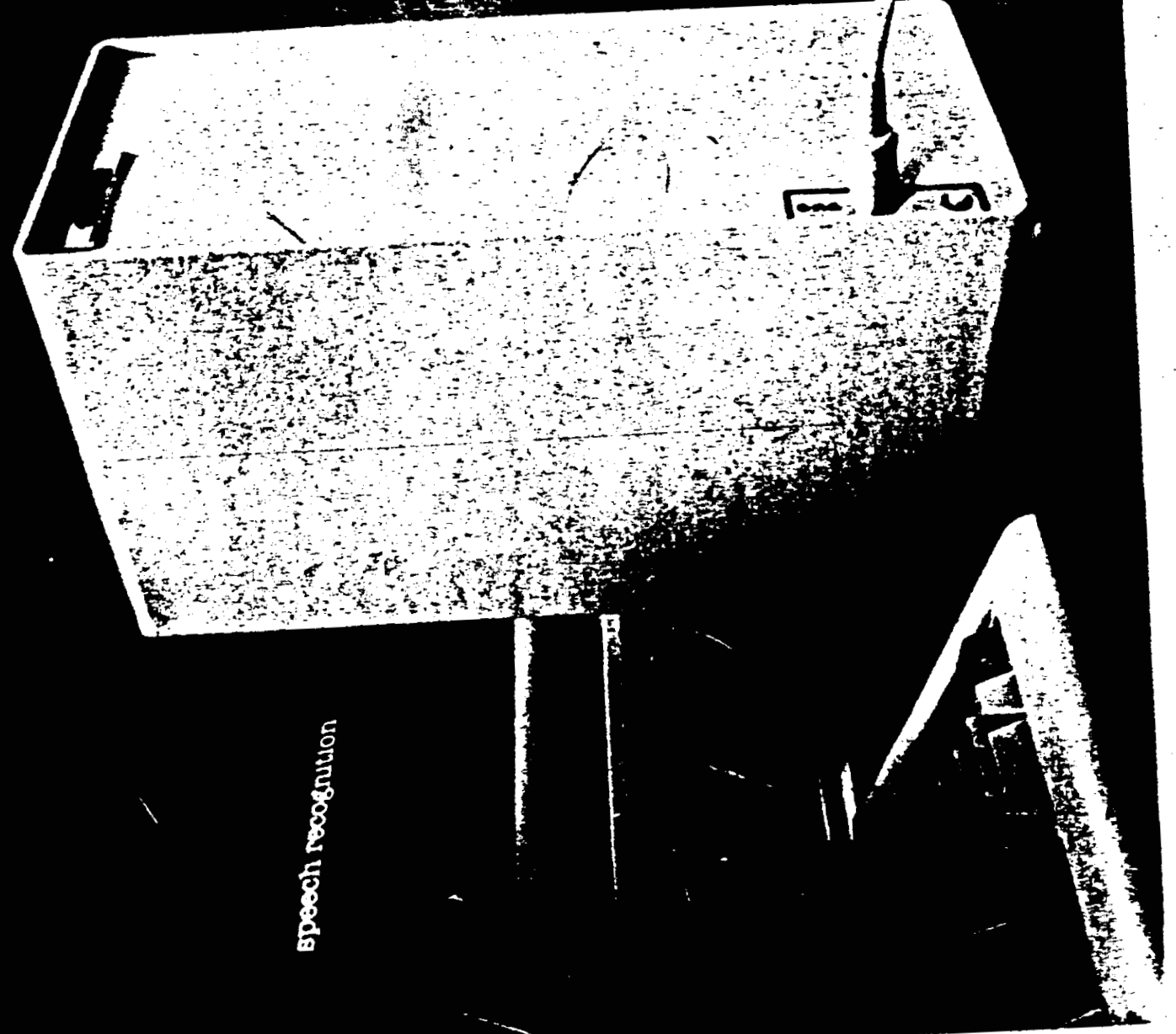


\section{TYPICAL IMAGE PROCESSING OPERATIONS}

The following are simplified explanations of some of the digital imaging processing terms.

\section{CONVOLUTION}

Convolution is used for spatial filtering and finding image features. The convolution operation replaces a pixel's value with the sum of that pixel's value and its neighbors values, each weighted by a factor. The weighting factors are called the convolution kernel. These kernels can take on various sizes, however the typical kernels are square arrays such as $3 \times 3,5 \times 5,7 \times 7$, etc. It is not necessary for the arrays to be square but a square one can be reduced to a linear or rectangular array by making the weighting factors zero for the row(s) or column(s) which are not desired. The arrays are usually of odd dimensions so that there is not a shift of the image and also so that the effect of the kernel can be symetric if desired. Convolution is computationally expensive, therefore, larger kernels significantly slow down image processing. Convolution result in a border of garbage around the inage edge where the kernel cannot be properly applied to the edge pixels. The larger the kernels are and the number of times the convolution is done results in larger borders.

\section{DIFFERENTIATION}

Differentiation can be used to sharpen the image. Differentiation quantifies the rate of change of the intensities of the image. Edges usually have significant intensity changes and they can be enhanced through differentation. In the continuous realm a gradient vector at any point is formed by taking the partial derivatives in $x$ and $y$ at that point. Since we are dealing with digital images there are a finite number of points. The magnitudes of the gradient vector at each of these points is approximated by sums of differences. These gradient magnitudes are generated by applying various difference operators to the digitized image at each point. These operators are small orthorgonal arrays. The common approximation of the gradient $g(x, y)$ is derived from the image $f(x, y)$ by taking the sum of the absolute values of the differences of the intensities between the current $x$ and $y$ position and the next position in the $x$ direction $(x+1)$ and the next position in the $y$ direction $(y+1)$. Mathematically

$$
g(x, y)=\operatorname{abs}[f(x, y)-f(x+1, y)]+\operatorname{abs}[f(x, y)-f(x, y+1)] .
$$

The difference operators in this case are:

$\begin{array}{rrrr}1 & -1 & 1 & 0 \\ 0 & 0 & -1 & 0\end{array}$




\section{ROBERTS FILTER}

The Roberts Edge Filter or Roberts Gradient is a variation of the gradient as described above. Mathematically

$$
g(x, y)=\operatorname{abs}[f(x, y)-f(x+1, y+1)]+\operatorname{abs}[f(x+1, y)-f(x, y+1)]
$$

The difference operators in the case are:

$\begin{array}{ccccc}1 & 0 & \text { and } & 0 & 1 \\ 0 & -1 & & -1 & 0\end{array}$

\section{PREWITT and SOBEL}

Prewitt and Sobel Edge operators are $3 \times 3$. There effect is to reduce the effects of noise by local averaging. The Sobel operators give larger weighting to the closer pixels than to the corner pixels.

Prewitt operators:

$\begin{array}{rrrrrrr}-1 & 0 & 1 & & 1 & 1 & 1 \\ -1 & 0 & 1 & \text { and } & 0 & 0 & 0 \\ -1 & 0 & 1 & & -1 & -1 & -1\end{array}$

Sobel operators:

$\begin{array}{rrrrrrr}-1 & 0 & 1 & & 1 & 2 & 1 \\ -2 & 0 & 2 & \text { and } & 0 & 0 & 0 \\ -1 & 0 & 1 & & -1 & -2 & -1\end{array}$




\section{LAPLACIAN}

The Laplacian is an edge detection operator. It is an approximation to the mathematical Laplacian, that is the second partial derivative of $f(x, y)$ with respect to $x$ and $y$.

The Laplacian has two disadvantages:

a.) directional information is not available, and

b.) it enhances noise.

One version of the discrete Laplacian is given by

$$
L(x, y)=f(x, y)-(1 / 4)[f(x, y+1)+f(x, y-1)+f(x+1, y)+f(x-1, y)] \text {. }
$$

This edge detection operator in kernel form is

$$
\begin{aligned}
& \begin{array}{lll}
0 & -1 & 0
\end{array} \\
& \begin{array}{lll}
-1 & 4 & -1
\end{array} \\
& \begin{array}{llll}
0 & -1 & 0
\end{array}
\end{aligned}
$$

Another Laplacian edge detection kernel is

$$
\begin{aligned}
& \begin{array}{lll}
-1 & -1 & -1
\end{array} \\
& \begin{array}{lll}
-1 & 8 & -1
\end{array} \\
& \begin{array}{llll}
-1 & -1 & -1
\end{array}
\end{aligned}
$$

Laplacian edge enhancement kernels have additional weighting at the center which retains the original image but enhances the edges. Here are two of these kernels:

$\begin{array}{rrrrrrr}0 & -1 & 0 & & -1 & -1 & -1 \\ -1 & 5 & -1 & \text { and } & -1 & 9 & -1 \\ 0 & -1 & 0 & & -1 & -1 & -1\end{array}$




\section{HOMOMORPHIC FILTERING}

An Image can be approximated by an 1llumination-reflectance mocel. The illumination is responsible for the dynamic range and in general is characterised by slow spatial variations. On the other hand contrast is a function of the reflectivity of objects and varies abruptly particularly at object boundaries. A homomorphic filter which will affect low and high spatial frequencies differently can effectivey compress the dynamic range and enhance the contrast simultaneously if the low frequencies are attenuated and the high frequencies amplified. This process is mathematically involved.

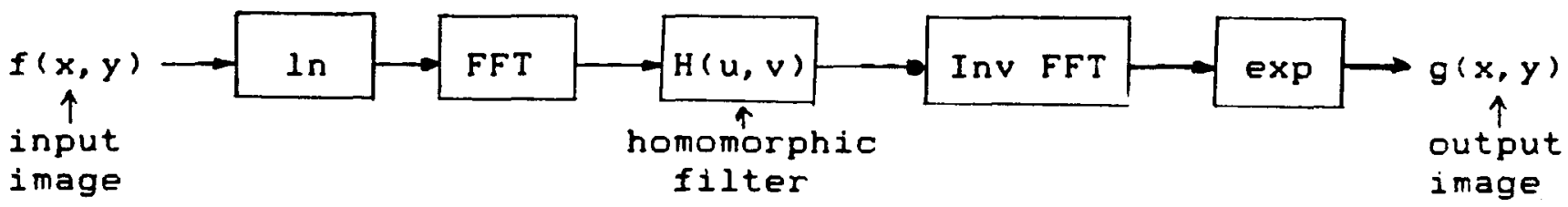

\section{TIME AVERAGING}

Averaging multiple noisy images will remove a large portion of the uncorrelated noise. The improvement will depend on the number of images averaged. This is useful for 1 mage acquisition where the original image is time invariant, such as is the case in digitizing radiographic images.

\section{NEIGHBORHOOD AVERAGING}

A pixel is replaced by a weighted average of its neighbors. This tends to remove noise, however, it also removes detail and causes edge blurring. Some averaging kernels are:

\begin{tabular}{|c|c|c|c|c|c|c|c|c|c|}
\hline & 0 & 1 & 0 & & & & 1 & 1 & 1 \\
\hline \multirow[t]{3}{*}{$1 / 5$} & 1 & 1 & 1 & . & & $1 / 9$ & 1 & 1 & 1 \\
\hline & 0 & 1 & 0 & & & & 1 & 1 & 1 \\
\hline & 1 & 1 & 1 & & & & 1 & 2 & 1 \\
\hline \multirow[t]{2}{*}{$1 / 10$} & 1 & 2 & 1 & . & and & $1 / 16$ & 2 & 4 & 2 \\
\hline & 1 & 1 & 1 & & & & 1 & 2 & 1 \\
\hline
\end{tabular}

MEDIAN FILTERING

A pixel is replaced by the middle value of its neighbors after they are numerically sorted. 